\title{
Myocardial perfusion imaging: a plus for coronary risk classification in diabetics
}

\author{
Albert Flotats \\ Published online: 31 January 2012 \\ (C) Springer-Verlag 2012
}

Type 2 diabetes mellitus is the most common form of diabetes worldwide ( $>90 \%)$. Long-term complications of diabetes include microvascular and macrovascular damage. Life expectancy of patients with diabetes remains substantially shorter than that of individuals without diabetes, mainly because of coronary heart disease (CHD) [1]. However, CHD risk in patients with diabetes is highly variable and difficult to stratify by conventional clinical markers [2, 3]. Moreover, the early identification of CHD in diabetics is difficult due to its often silent or atypical presentation in this population. As a result, CHD in diabetics may be diagnosed several years after its onset, when the disease is in an advanced stage, frequently in the form of myocardial infarction (MI) or sudden cardiac death. To improve outcomes, common CHD risk factors should be assessed at least annually, along with the presence of micro- or macroalbuminuria, and be actively managed $[4,5]$.

The increasing prevalence of diabetes and the associated high CHD risk raises the question as to the need to develop noninvasive approaches to detect the patients with diabetes at the highest risk of CHD. This strategy could identify higher risk diabetics in whom coronary revascularization may improve the outcome beyond that achieved by currently recommended medical management. Stress/rest myocardial

\footnotetext{
A. Flotats

Nuclear Medicine Department,

Universitat Autònoma de Barcelona,

Barcelona, Spain

A. Flotats $(\square)$

Nuclear Medicine Department,

Hospital de la Santa Creu i Sant Pau,

Sant Antoni M. Claret, 167,

08025 Barcelona, Spain

e-mail: aflotats@santpau.cat
}

perfusion imaging (MPI) is effective in detecting CHD and predicting adverse cardiac events in diabetic patients [6, 7]. Nevertheless, the clinical utility of MPI in diabetics is debated due to the small number of prospective and outcome studies, along with some epidemiological and cost-effectiveness data.

It has been reported that routine screening of asymptomatic diabetics who have a normal ECG does not have clinical benefit and that cardiac outcomes are essentially equal (and very low) in screened and unscreened patients (despite abnormal MPI in $>20 \%$ of these patients) [8]. In addition, according to the large multicentre Bypass Angioplasty Revascularization Investigation 2 Diabetes trial (BARI 2D), optimal intensive medical therapy provides equal outcomes to revascularization in patients with diabetes [9]. In this randomized trial, only patients who had undergone coronary bypass surgery had significantly reduced major cardiac events, compared with those assigned in the intensive medical therapy group, but no difference in mortality was recorded. Therefore, only those diabetics with evidence of myocardial ischaemia and extensive multivessel disease would benefit from prompt surgical revascularization, mainly because of a lower rate of nonfatal MI. It is noteworthy that the majority of the patients enrolled had symptoms and all of them had stable CHD [9]. There is also some evidence that silent myocardial ischaemia may reverse over time with optimized medical treatment, adding to the controversy concerning aggressive screening strategies [10]. Finally, it has been reported that even asymptomatic diabetics with intermediate/high CHD risk have a low annual cardiac event rate $(<1 \%)$, which is not affected by routine screening for inducible ischaemia [11].

Since screening for CHD in asymptomatic patients does not improve outcomes as long as CHD risk factors are treated, it is not routinely recommended by professional organizations or guidelines for appropriate use of cardiac 
radionuclide imaging $[4,5,12]$. Testing for ischaemia is recommended in selected individuals with a strong suspicion of a high CHD risk, i.e. diabetics with typical or atypical cardiac symptoms and those with an abnormal resting ECG.

In this issue of the journal, Acampa et al. present the results of the inDucible Ischemia by Stress MPI (IDIS) trial [13]. This is a prospective multicentre trial performed in diabetic patients referred for MPI with the aim of determining whether the addition of stress/rest SPECT MPI to traditional clinical risk factors improves the classification of risk for the prediction of cardiac events during a long-term follow-up. To this purpose, the authors analysed a collected database of 822 consecutive patients with a history of type 2 diabetes of $\geq 5$ years duration using two different Cox models of proportional hazards. Model 1 used traditional CHD risk factors in diabetics and ECG stress test data and model 2 used these variables plus MPI. The prevalence of abnormal MPI was $50 \%$. After a 5-year follow-up period, 148 events occurred (18\% cumulative event rate), including cardiac death $(n=31)$, MI $(n=30)$ and unstable angina requiring revascularization $(n=87)$. Patients with events had significantly higher clinical CHD risk, lower LVEF and a higher prevalence of an abnormal MPI ( $77 \%$ vs. $44 \%)$. Clinical CHD risk and abnormal MPI were independent predictors of events in both the univariate and multivariate analysis. MPI provided incremental prognostic information over clinical CHD risk and LVEF. The annual event rate according to the clinical CHD risk and MPI increased progressively as the summed stress score increased in all risk categories (range 1.8-13.6\%). Reclassification of a patient's risk was defined as correct or incorrect according to outcome, and the net reclassification improvement (NRI, i.e. the difference between correct and incorrect reclassification) was computed to compare the distribution of risk in the two models. Model 2 improved risk prediction compared to model 1 , providing a reclassification to a lower risk category in $3 \%$ of the patients, and to a higher risk category in $37 \%$ of the patients. Among patients at intermediate clinical risk ( $3 \%$ to $<5 \%$ per year), $25 \%$ were reclassified to a lower risk and $53 \%$ to a higher risk. This latter group of patients experienced the majority of events (71\%) among those initially classified at intermediate risk. The authors also used the method of NRI to estimate the incremental cost for stress/rest MPI over an outpatient visit with an ECG stress test. They found that the cost per NRI for a MPI scan decreased by $40 \%$ when the test was indicated only in diabetics at intermediate clinical risk.

On the basis of this analysis the authors conclude that inclusion of stress/rest MPI data in a prediction model based on traditional risk factors and ECG stress data can improve CHD risk classification in diabetics.
This improvement is substantially higher in the subgroup of diabetics at intermediate clinical risk. Patients in this category appear to be those who would benefit the most from the addition of MPI [13].

The paper is interesting and covers a topic of high clinical and economic relevance, supporting the value of MPI for risk stratification of diabetic patients. The basis of testing for risk stratification is that only those patients who can be successfully further stratified in a cost-effective manner are appropriate for the test [14]. In this sense, the results of the IDIS trial are in accordance with the concept that MPI is cost-effective only when applied to intermediate and high risk patients, regardless of its success for risk stratification in other patient populations [15]. Furthermore, these results support the additional role of MPI in conventional stress testing for accomplishing the specific objective of minimizing the number of patients who are still classified as intermediate risk after the completion of testing. However, the results obtained cannot be compared to those of the DIAD study/substudies $[2,8,10,11]$, mainly because of differences in the underlying risk of the patients studied, i.e. the prevalence of abnormal MPI in the IDIS trial was $50 \%$ vs. $22 \%$ in the DIAD study. Furthermore, all patients enrolled in the DIAD study had type 2 diabetes, no symptoms suggesting CHD, normal resting ECG and no prior cardiac stress testing. On the other hand, the IDIS trial did not exclude patients with a history of CHD, and actually about $35 \%$ of patients had previous MI. In addition, the trial included symptomatic and asymptomatic diabetic patients with different prescan likelihoods of ischaemia, although detailed information on patients' symptoms or the proportion of insulin-dependent patients (who are known to be at higher CHD risk [6]) is not available. Along with this remark, Acampa et al. [13] appropriately make reference to the inclusion of an increased number of patients with previous MI, which may have introduced a selection bias (large number of patients with higher CHD risk). However, as the authors state, the high prevalence of CHD found in autopsy studies in diabetics without previous suspected CHD supports the methodology used. Furthermore, their strategy is in accordance with current recommendations, which propose MPI in diabetics with a strong suspicion of high-risk CHD. Another potential limitation of the study, recognized by the authors as well, is that the MPI results were revealed to the participants and their physicians. This may have affected patient management, especially referral for revascularization, thus affecting the observed patients' risk in proportion to the imaging results (post-test referral bias) [14]. However, the analysis of Acampa et al. [13] shows that MPI provides independent prognostic information even when only cardiac death or MI (and not revascularization) were considered as end-points, which gives relevance to their study 
and reinforces the benefit of risk assessment by MPI in diabetics, in accordance with previous reports $[6,7]$.

Originally, the IDIS trial was conceived to estimate the time to predefined risk and level of risk at specific time intervals during follow-up, in addition to the evaluation of the prevalence of inducible ischaemia and the determination of the number of patients who were reclassified with respect to their risk of adverse outcomes by the addition of MPI data to pretest information [16]. By evaluating how the risk changes over time as a function of the patients' characteristics and test results, the aim of the investigators was to stipulate the warranty period before retesting after a normal MPI. Unfortunately, this relevant issue was not referred to in the final published data of the trial.

Given the diversity of pretest CHD risk in diabetics, and the availability of different techniques that provide early identification of atherosclerosis (coronary artery calcium score, CAC) and information on plaque composition (multidetector CT angiography, MDCTA), a stepwise screening strategy has been proposed, starting with CAC or MDCTA followed by MPI when moderate coronary disease is present $[17,18]$. Actually, these methods have shown prognostic value in patients with diabetes $[19,20]$ and their inclusion in the IDIS trial would have been appealing. In spite of this, the IDIS trial [13] is a well-respected and dignified study, in full accord with the concept of "Idis", which according to Wikipedia corresponds to a divine female of Germanic mythology, and means "well-respected and dignified woman". The results, obtained from a quite large-scale multicentre registry of patients in the modern era of wide endorsement and implementation of primary cardiac prevention measures, support the incremental value of SPECT MPI for the assessment of CHD risk in diabetics, especially in those at intermediate clinical risk of CHD. Therefore, a scheme that includes such data would appear to be useful in refining the risk estimation in diabetics referred for MPI. Additional prospective multicentre studies are needed to determine if, under optimized intensive medical therapy, a combination of different imaging modalities and surrogate markers is needed to identify patients at most CHD risk.

\section{References}

1. Kannel WB, McGee DL. Diabetes and cardiovascular disease. The Framingham study. JAMA. 1979;241:2035-8.

2. Wackers FJ, Young LH, Inzucchi SE, Chyun DA, Davey JA, Barrett EJ, et al. Detection of silent myocardial ischemia in asymptomatic diabetic subjects: the DIAD study. Diabetes Care. 2004;27:1954-61.
3. Scognamiglio R, Negut C, Ramondo A, Tiengo A, Avogaro A. Detection of coronary artery disease in asymptomatic patients with type 2 diabetes mellitus. J Am Coll Cardiol. 2006;47:65-71.

4. American Diabetes Association. Executive summary: standards of medical care in diabetes - 2011. Diabetes Care 2011;34 Suppl 1: S4-10.

5. Graham I, Atar D, Borch-Johnsen K, Boysen G, Burell G, Cifkova $\mathrm{R}$, et al. European guidelines on cardiovascular disease prevention in clinical practice: executive summary. Fourth Joint Task Force of the European Society of Cardiology and other societies on cardiovascular disease prevention in clinical practice (constituted by representatives of nine societies and by invited experts). Eur J Cardiovasc Prevent Rehabil 2007;14 Suppl 2:E1-40.

6. Berman DS, Kang X, Hayes SW, Friedman JD, Cohen I, Abidov A, et al. Adenosine myocardial perfusion singlephoton emission computed tomography in women compared with men. Impact of diabetes mellitus on incremental prognostic value and effect on patient management. J Am Coll Cardiol. 2003;41:1125-33.

7. Zellweger MJ, Hachamovitch R, Kang X, Hayes SW, Friedman JD, Germano G, et al. Prognostic relevance of symptoms versus objective evidence of coronary artery disease in diabetic patients. Eur Heart J. 2004;25:543-50.

8. Young LH, Wackers FJ, Chyun DA, Davey JA, Barrett EJ, Taillefer R, et al. Cardiac outcomes after screening for asymptomatic coronary artery disease in patients with type 2 diabetes: the DIAD study: a randomized controlled trial. JAMA. 2009;301:1547-55.

9. BARI 2D Study Group; Frye RL, August P, Brooks MM, Hardison RM, Kelsey SF, MacGregor JM, et al. A randomized trial of therapies for type 2 diabetes and coronary artery disease. N Engl J Med. 2009;360:2503-15.

10. Wackers FJ, Chyun DA, Young LH, Heller GV, Iskandrian AE, Davey JA, et al. Resolution of asymptomatic myocardial ischemia in patients with type 2 diabetes in the Detection of Ischemia in Asymptomatic Diabetics (DIAD) study. Diabetes Care. 2007;30:2892-8.

11. Bansal S, Wackers FJ, Inzucchi SE, Chyun DA, Davey JA, Staib $\mathrm{LH}$, et al. Five-year outcomes in high-risk participants in the Detection of Ischemia in Asymptomatic Diabetics (DIAD) study: a post hoc analysis. Diabetes Care. 2011;34:204-9.

12. Hendel RC, Berman DS, Di Carli MF, Heidenreich PA, Henkin RE, Pellikka PA, et al. ACCF/ASNC/ACR/AHA/ASE/SCCT/ SCMR/SNM 2009 Appropriate Use Criteria for Cardiac Radionuclide Imaging: A Report of the American College of Cardiology Foundation Appropriate Use Criteria Task Force, the American Society of Nuclear Cardiology, the American College of Radiology, the American Heart Association, the American Society of Echocardiography, the Society of Cardiovascular Computed Tomography, the Society for Cardiovascular Magnetic Resonance, and the Society of Nuclear Medicine. J Am Coll Cardiol. 2009;53:2201-29.

13. Acampa W, Petretta M, Evangelista L, Daniele S, Xhoxhi E, De Rimini ML, et al. Myocardial perfusion imaging and risk classification for coronary heart disease in diabetic patients. The IDIS study: a prospective, multicentre trial. Eur J Nucl Med Mol Imaging. 2011. doi:10.1007/s00259-011-1983-x

14. Hachamovitch R, Di Carli MF. Methods and limitations of assessing new noninvasive tests: Part II: Outcomes-based validation and reliability assessment of noninvasive testing. Circulation. 2008;117:2793-801.

15. Hachamovitch R, Hayes SW, Friedman JD, Cohen I, Berman DS. Stress myocardial perfusion single-photon emission computed tomography is clinically effective and cost effective in risk stratification of patients with a high likelihood of coronary artery disease (CAD) but no known CAD. J Am Coll Cardiol. 2004;43:200-8. 
16. Petretta M, Acampa W, Evangelista L, Daniele S, Ferro A, Cuocolo A. Impact of inducible ischemia by stress SPECT in cardiac risk assessment in diabetic patients: rationale and design of a prospective, multicenter trial. J Nucl Cardiol. 2008;15:100-4.

17. Anand DV, Lim E, Lahiri A, Bax JJ. The role of non-invasive imaging in the risk stratification of asymptomatic diabetic subjects. Eur Heart J. 2006;27:905-12.

18. Bax JJ, Bonow RO, Tschöpe D, Inzucchi SE, Barrett E; Global Dialogue Group for the Evaluation of Cardiovascular Risk in Patients With Diabetes. The potential of myocardial perfusion scintigraphy for risk stratification of asymptomatic patients with type 2 diabetes. J Am Coll Cardiol. 2006;48: 754-60.

19. Raggi P, Shaw LJ, Berman DS, Callister TQ. Prognostic value of coronary artery calcium screening in subjects with and without diabetes. J Am Coll Cardiol. 2004;43:1663-9.

20. Van Werkhoven JM, Cademartiri F, Seitun S, Maffei E, Palumbo A, Martini C, et al. Diabetes: prognostic value of CT coronary angiography - comparison with a nondiabetic population. Radiology. 2010;256:83-92. 